\title{
Wirbelsäulenverletzungen mit Querschnittlähmung - aktueller Stand der Behandlung
}

\author{
Rainer Abel, Hans Jürgen Gerner
}

\begin{abstract}
Zusammenfassung
Verletzungen der Wirbelsäule mit Querschnittlähmung sind schwere Verletzungen mit ausgedehnten Begleitschädigungen. Ihre Versorgung ist komplex, die Indikation zur notfallmäßigen operativen Stabilisation nur auf Einzelfälle beschränkt. Die Versorgung sollte deshalb in dafür spezialisierten Zentren und von Teams mit ausreichender Erfahrung erfolgen. Eine 24 Std. und 7 Tage die Woche besetzte Hotline an der BG-Unfallklinik Hamburg, (Tel. 040/73062604) gibt Auskunft und Hilfestellung und nennt die übernahmebereiten Zentren mit freier Bettenkapazität und Versorgungsmöglichkeit.
\end{abstract}

\section{Einleitung}

Die traumatische Querschnittlähmung ist ein lange bekanntes und auch in der Antike schon beschriebenes Krankheitsbild [7]. Diese Berichte sprechen von der Aussichtslosigkeit der Prognose und dem zu erwartenden Siechtum bis zum Tod des Verletzten.

Weitere Berichte von der Behandlung Querschnittgelähmter, die auch die ersten Berichte über das operative Versorgen und Vorgehen beinhalten, finden Ende des 19. Jahrhunderts Eingang in die chirurgische Literatur [9]. Allerdings sind die Ergebnisse der operativen Versorgung sowie die Indikationsstellung noch so, dass Wagner und Stolper 1898 feststellen: „... so kommt man zu dem wenig

OP-JOURNAL 2001; 17: 172-176

(C) Georg Thieme Verlag Stuttgart · New York trostvollen Resultat, dass bei weitem häufiger kühner Wagemuth als Objektivität und wissenschaftliche Ueberzeugung bisher den Operateuren das Messer in die Hand gedrückt hat.“

Eine Wende hat die Versorgung von Querschnittgelähmten in Europa genommen, als Ludwig Guttmann [6], beauftragt wurde, für die britischen querschnittgelähmten Verletzten des 2. Weltkrieges in Stoke Mandeville ein Versorgungszentrum aufzubauen. Er konnte nachweisen, dass die üblicherweise zum Tode führenden Komplikationen wie infizierte Druckgeschwüre und chronisches Nierenversagen vermieden werden können. Guttmann und seine Schule vertraten aufgrund der vor und von ihnen gemachten schlechten Erfahrungen mit der operativen Versorgung von Querschnittgelähmten strikt die Auffassung „non touch“= keine OP - im Sinne einer strengsten Indikationsstellung.

Insbesondere die Vorstellung, durch eine operative Intervention mit der Wiederherstellung des Spinalkanals eine Verbesserung der neurologischen Prognose erzielen zu können, hat sich immer wieder als falsch erwiesen. Auch eine Übersichtsarbeit aus neuester Zeit [2] bestätigt den bis heute fehlenden Nachweis einer Verbesserung der neurologischen Funktion durch Dekompression und Wiederherstellung des Spinalkanals.

Trotzdem hat im Zuge der Entwicklung verbesserter Implantate und des immer besseren Verständnisses der biomechanischen Prinzipien der Wirbelsäule auch die operative Versorgung von Wirbelsäulenfrakturen Eingang in die Behandlungsstrategie gefunden. Allerdings müssen sich die Ergebnisse der operativen Versorgung immer noch an den gut dokumentierten Ergebnissen der konservativen Behandlung [4] messen lassen.

\section{Inzidenz der Querschnittlähmung}

Seit 1976 werden fortlaufend die Daten aus den deutschen Spezialzentren für Querschnittgelähmte zentral gesammelt. Bis Ende 2000 wurden rund 29000 Patienten mit einer frischen Querschnittlähmung erfasst. $71 \%$ erlitten eine traumatische, 29\% eine nicht traumatische Querschnittlähmung. 32\% der Unfälle ereigneten sich im Verkehr, 13\% bei der Arbeit. Im langfristigen Trend ist eine Zunahme des Anteils der nicht traumatischen und ein Rückgang der verkehrsbedingten Querschnittlähmungen erkennbar.

Pro Jahr beträgt die Zahl der in den Spezialzentren (24, davon 18 für Akutversorgung) versorgten Frischverletzten etwa 1600 (Abb.1).

\section{Indikation für die operative Versorgung von Wirbelsäulen- verletzungen bei frischen Querschnittlähmungen}

Das Ausmaß der Querschnittlähmung bestimmt sich in der Unfallsekunde. Die Kraft mit der Fragmente zu diesem Zeitpunkt in den Spinalkanal gepresst werden und die Position, die in diesem Augenblick erreicht wird, sind für das Ausmaß der Schädigung des Rückenmarkes verantwortlich. Bereits die durch gezielte und schonende Lagerung „postural reduction“ [6] erreichte Spontanreposition unterscheidet sich deutlich von der maximalen Dislokation der frakturierten Knochenanteile. Außerdem sind die verbleibenden Druckkräfte, die auf das Rückenmark wirken, im Bereich weniger Kilopascal, während sie zum Zeitpunkt der Fraktur einige hundert Kilopascal erreichen [2].

Aus diesen Gründen ist die einzige Indikation - außer der offenen Wirbelsäulenverletzung -, die zur Notfallversorgung zwingt, eine Querschnittlähmung, die sich verschlechtert. Primär komplette 


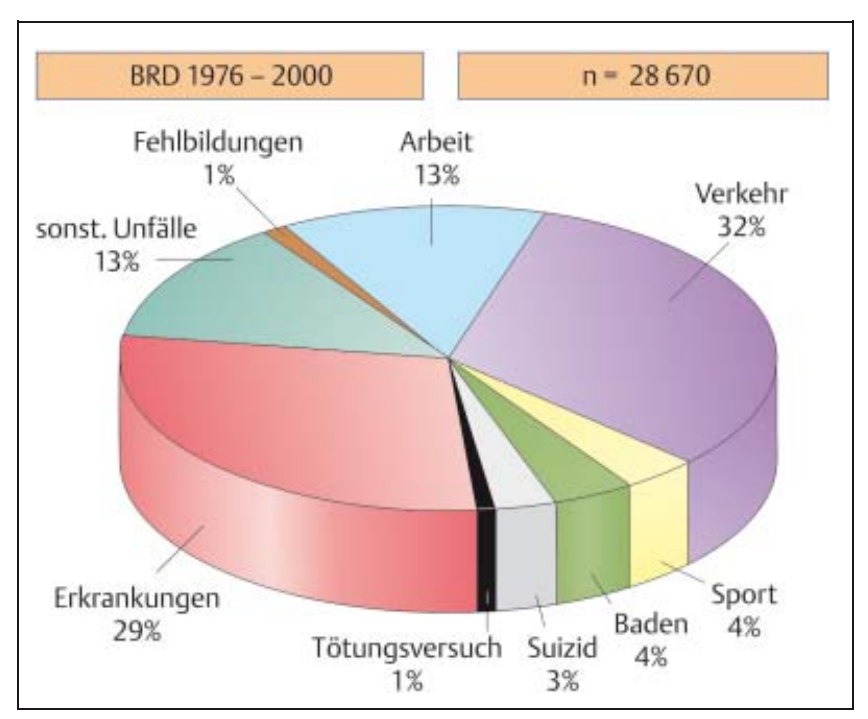

Abb.1 Grafische Darstellung des Anteils der Lähmungsursachen an den in den deutschen Querschnittzentren erfassten Patienten.

Querschnittlähmungen haben durch eine sofortige Notfalloperation keine bessere Prognose.

Bei primär inkompletten Rückenmarkverletzungen verhindert eine frühestmögliche Stabilisierung der Fraktur mit dem Ziel „Ruhe im Verletzungsbereich“ eine Sekundärschädigung durch Instabilität.

Die Erfahrung und die Literatur der vergangenen Jahrzehnte zeigen, dass ein Großteil der Wirbelsäulenfrakturen konservativ zur Ausheilung und Stabilität gebracht werden kann. Allerdings bedarf es dazu bei einer traumatischen Querschnittlähmung eines Zeitraums bis zu 3 Monaten, in denen der Verletzte nicht mobilisiert werden kann. Auch aus diesem Grund wird heute die überwiegende Anzahl von Frakturen operativ stabilisiert.

Ziel der operativen Versorgung ist die Wiederherstellung der ursprünglichen Wirbelsäulenform, die bessere und frühzeitige Belastbarkeit sowie die Wiederherstellung des Spinalkanals durch ein an der Brust- und Lendenwirbelsäule meist kombiniertes ventral/dorsales Vorgehen. Üblicherweise werden von dorsal winkelstabile Instrumentarien eingesetzt (z.B. Fixateur interne), die eine sofortige postoperative Mobilisation ermöglichen. Die Fusionsstrecke muss so kurz wie möglich gehalten werden. Großzügiges Überbrücken mehrerer gesunder Wirbelsäulensegmente durch eine dorsale Instrumentation ist nach unserer Auffassung kontraindiziert.
Der wesentliche Grund durch das operative Vorgehen auch den Spinalkanal wieder herzustellen besteht nicht in der Hoffnung auf eine günstige Beeinflussung der unmittelbaren Lähmungsprognose. Vielmehr haben neuere, auch eigene Arbeiten [1] gezeigt, dass verbleibende Einengungen und Knickbildungen offenbar über eine chronische Fehlbelastung des Myelons zu Sekundärschäden führen, die sich erst Jahre später bemerkbar machen. Dazu gehört in erster Linie die sog. posttraumatische Syringomyelie. Es handelt sich dabei um eine meist höhlenförmige Aufweitung des Rückenmarkes, die von der Läsionsstelle nach kranial und kaudal fortschreitet und zu katastrophalen sekundären Lähmungsverschlechterungen, oft Jahre nach dem eigentlichen Unfall, führen kann (Abb. 2).

Für eine optimale Primärversorgung von Wirbelfrakturen mit neurologischem Defizit ist die Indikationsstellung und die richtige Zeitplanung besonders wichtig. Diese Wirbelsäulenverletzungen müssen so bald wie möglich von einem erfahrenen Team operiert werden. Dabei ist an der Brust- und Lendenwirbelsäule die primär dorsale Stabilisierung zunächst oft ausreichend.

Ein Teil der Verletzten hat eine ausgedehnte Lungenkontusion, viele sind mehrfach verletzt, weitere Verletzte haben retroperitoneale Blutungen. Wir sehen keinen Grund, das bei dem Trauma erzeugte Verletzungsmuster durch ein notfallmäßiges Vorgehen ventral an der Wirbelsäule zu verschlechtern.

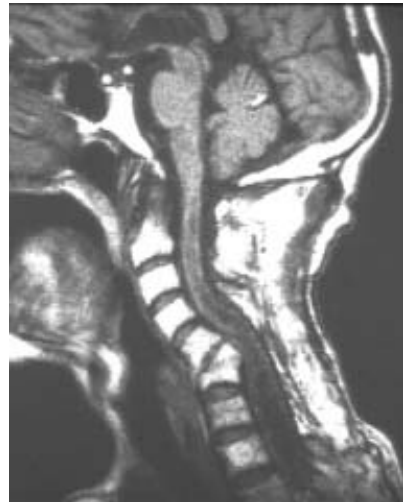

Abb.2 Typische Syringomyelie mit langstreckiger zystischer Aufweitung zentral im Rückenmark bei in Fehlstellung und mit Einengung des Spinalkanals ausgeheilter C5/6Luxationsfraktur.

Leider sehen wir immer wieder die Folgen heroischer Eingriffe in den nicht für die Spezialversorgung eingerichteten Kliniken, aus denen dann nach einigen Wochen die Patienten mit insuffizient behandelter Blasenlähmung, Darmlähmung, Druckgeschwüren etc. verlegt werden.

Prognostisch entscheidend ist nicht der Zeitpunkt der Frakturversorgung sondern die sichere Präventation und Beherrschung aller Komplikationen einer Querschnittlähmung.

\section{Frakturlokalisation und Typen}

Vor der Indikationsstellung ist die Übereinstimmung von neurologischem Befund und festgestellter Wirbelsäulenverletzung zu sichern. Die erste Forderung ist deshalb immer, die neurologische Läsionshöhe festzustellen. Diese muss zu der Frakturhöhe passen. Nicht selten kommt es zu Kombinationsverletzungen, wobei die lähmungsverursachende Fraktur übersehen oder falsch eingeschätzt wird.

Die Beurteilung der Operationswürdigkeit einer Wirbelverletzung hinsichtlich der zu erwartenden Ausheilungsaussichten und Stabilität wird durch verschiedene Klassifikationssysteme unterstützt [8].

\section{Halswirbelsäule}

Verletzungen der oberen Halswirbelsäule führen aufgrund des hier sehr weiten Spinalkanals seltener zu Rückenmarkverlet- 


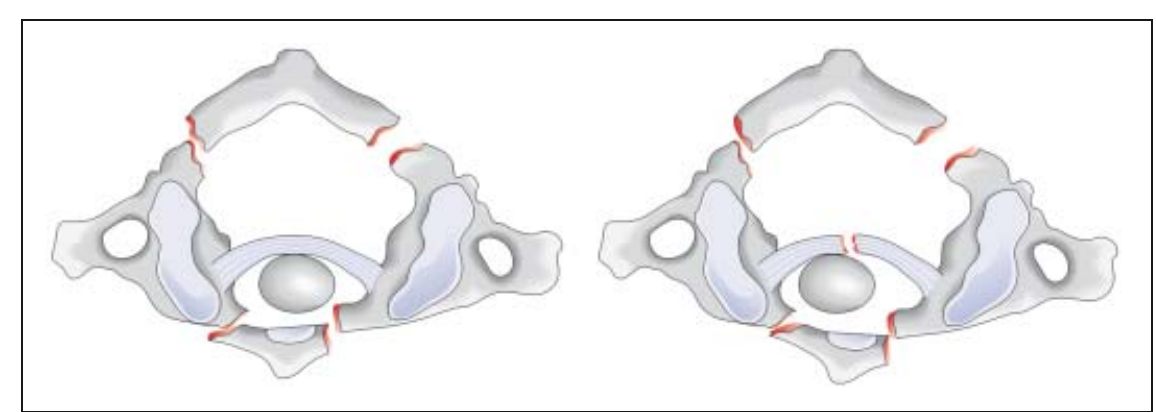

Abb. 3 Schematische Darstellung von Jefferson-Frakturen mit (instabil) und ohne (stabil) Ruptur des Lig. transversum.

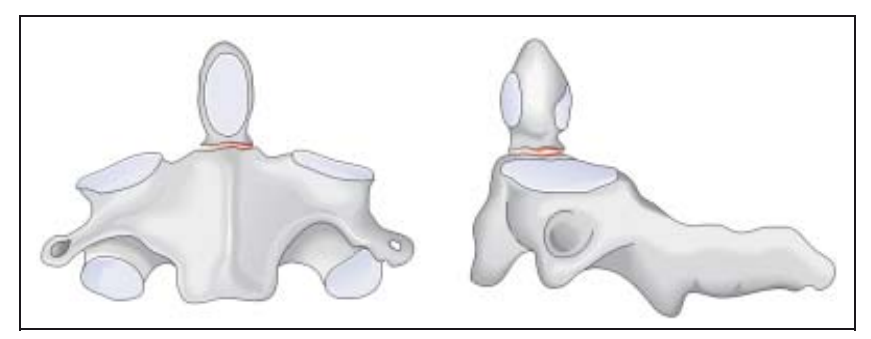

Abb. 4 Densfraktur vom Typ D‘Alonzo II. Densfraktur mit der gößten Pseudarthroserate.

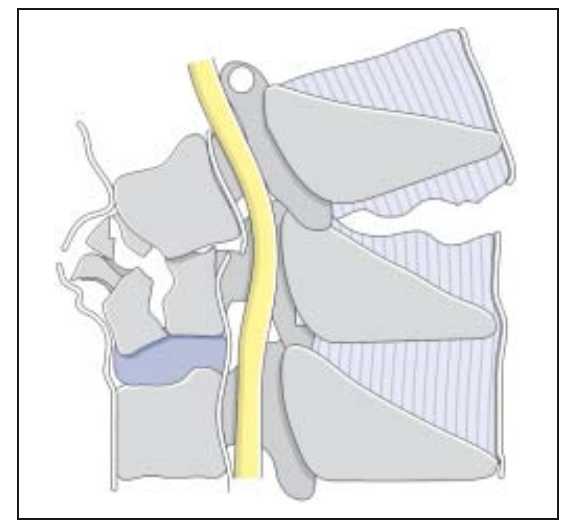

Abb. 5 Teardrop-Fraktur. Distraktions-Hyperflexions-Verletzung der HWS.

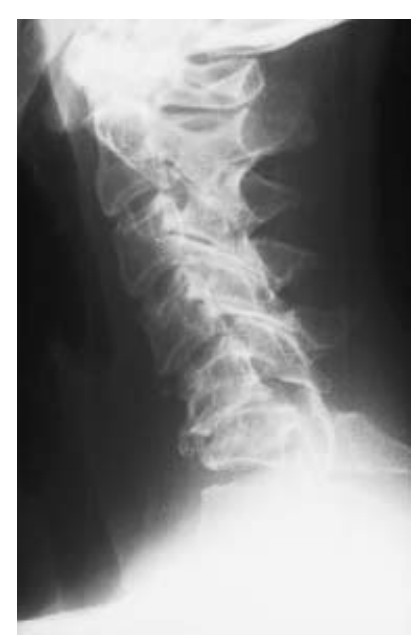

Abb. 6 Verhakte Luxationsfraktur C5/6. Deutlich ist die „reitende“ Stellung der Facetten zu erkennen. durch Hyperflektion, Hyperextension und Scherung. Eine Sonderform ist die so genannte Teardrop fracture, bei der es sich um eine Distraktions-Hyperflektionsverletzung handelt (s. Abb.5). Hier wird die ventrale Wirbelkörperkante abgerissen und meiselartig in den darunter liegenden Wirbelkörper hineingepresst.

Vor der operativen Versorgung muss feststehen, ob sich bei der Verletzung die dorsalen Facettengelenke in Luxationsstellung einseitig oder beidseitig verhakt haben. In diesen Fällen, kann eine geschlossene Reposition nach Anlage einer Crutchfield-Klammer unter kontinuierlichem Zug mit etwa 10\% des Körpergewichtes Erfolg haben. Es kann auch eine geschlossene Reposition in Narkose möglich sein, so lange die Verletzung frisch ist. Besteht eine nicht zu reponierende Luxation der Facettengelenke, muss zunächst über einen dorsalen Zugang eine teilweise Resektion der Facetten so vorgenommen werden, dass die Fraktur reponiert werden kann. In diesem Fall wird üblicherweise durch den dorsalen Zugang auch eine Osteosynthese entweder mittels Schrauben-Stab-System oder Cerclagen vorgenommen. Verletzungen der mittleren und unteren Halswirbelsäule werden von ventral mit einem PlattenSchrauben-System osteosynthetisch versorgt. Dabei werden die zerstörten Bandscheiben ausgeräumt und in ein stabiles Knochenlager ein kortikaler Beckenspan impaktiert. Es ist darauf hinzuweisen, dass sich die Versorgung einer Halswirbelfraktur mit einer teilweisen oder gänzlichen Zerstörung der dorsalen Bandstrukturen wesentlich von der ventralen Versteifung (z.B. Cloward oder Robinson) unterscheidet, wie sie nach Ausräumung einer isoliert geschädigten Bandscheibe (degenerativ oder Prolaps) vorgenommen wird. Wird dies missachtet, kann es z. B. (Abb.7 a u. b) zu einer unbeabsichtigten Distraktion der Halswirbelsäule mit unter Umständen bleibender Instabilität kommen.

Abschließend ist noch zu beachten, dass der Zugang zur Wirbelsäule nicht die Möglichkeit für eine sekundäre Tracheotomie bei lähmungsbedingter Ateminsuffizienz und Entwöhnungsproblemen vom Respirator beeinträchtigen sollte.

\section{Brust- und Lendenwirbelsäule}

Für die Klassifikation von Verletzungen an der Brust- und Lendenwirbelsäule hat sich in den letzten Jahren die von Ma-
Für Frakturen der mittleren und unteren Halswirbelsäule existiert kein allgemein anerkanntes Klassifikationsschema. Sie werden nach dem Unfallmechanismus eingeteilt. Man unterscheidet axiale Kompressionsfrakturen, Verletzungen 


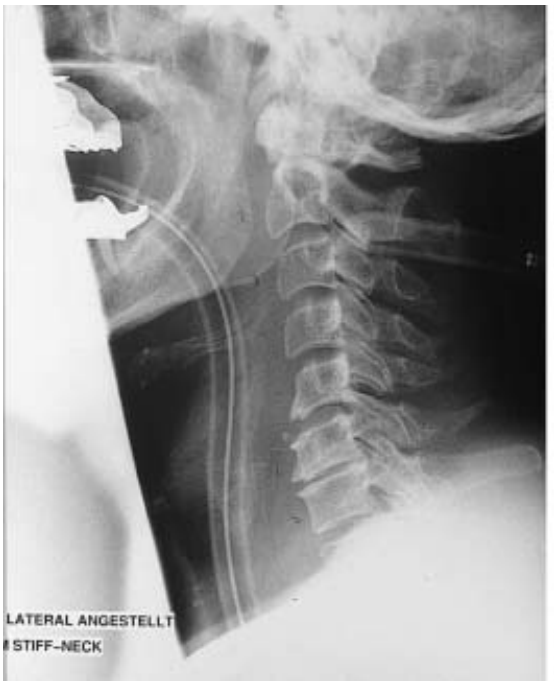

Abb.7a u. b In a ist die seitliche Röntgendarstellung der HWS nach Distraktionsverletzung mit initial kompletter Querschnittlähmung unterhalb C4/5 festgehalten. Gute spontane Reposition. b zeigt den Befund nach ventraler Interposition eines inadäquat

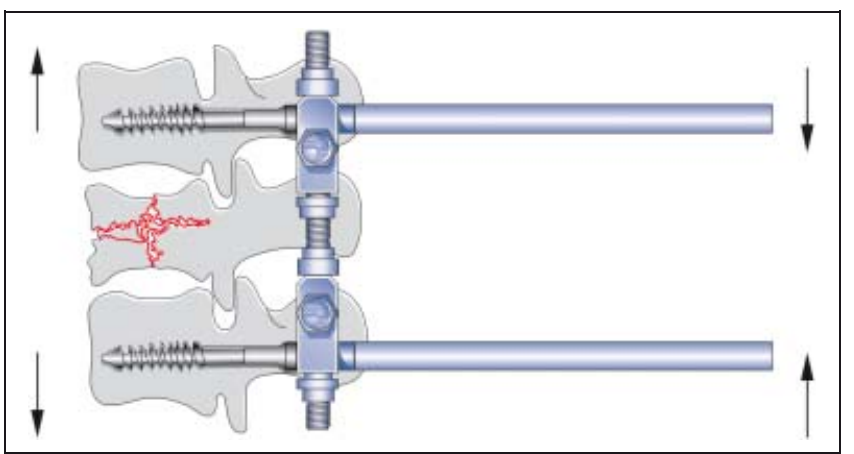

Abb. 8 Rotationszentren (Drehachsen) bei der Reposition von Wirbelfrakturen durch ein dorsales Implantat. Abbildung nach Dick [3].

gerl et al [8] vorgeschlagene Klassifikation durchgesetzt. Man unterscheidet zwischen Kompressionsverletzungen, Flektions-Distraktionsverletzungen und Frakturen mit zusätzlicher Rotationsund/oder Translationskomponente.

Bis auf wenige Ausnahmen handelt es sich bei Verletzungen der Wirbelsäule mit Querschnittlähmung um instabile Frakturen, meistens mit Rotationskomponente. In wenigen Ausnahmefällen kommt es bei einer an sich stabilen Wirbelsäulenverletzung zu einer Querschnittlähmung durch eine Contusio spinalis.

Die Versorgung erfolgt bei uns primär von dorsal transpedikulär durch winkelstabile Schrauben-Stab-Systeme [5]. Man sollte Titan-Implantate verwenden, um die sekundär oft notwendigen kernspintomographischen Verlaufskontrollen, insbesondere bei Lähmungsverände- rungen, Auftreten von Spastik, Syringomyelie etc. nicht zu beeinträchtigen. Die meisten Frakturen, insbesondere der unteren Brustwirbelsäule sowie der oberen Lendenwirbelsäule können bereits durch eine Lagerung im Durchhang suffizient reponiert werden. Hier muss das verwendete Implantat-System lediglich eine sichere Fixierung in reponierter Stellung gewährleisten. Frakturen der mittleren und oberen Brustwirbelsäule stehen jedoch manchmal in einer verhakten Fehlstellung, wobei die Rippen und ihre Verbindungen zu den Wirbelkörpern bei der Reposition zusätzlich stören. In diesen Fällen empfiehlt sich die Verwendung eines Implantatsystems, das über Repositionshilfen verfügt.

Von Dick wurde das Prinzip der Reposition einer Wirbelsäulenfraktur von dorsalbeschrieben [3]. Wichtig ist hierbei das Wissen um den Drehpunkt bei der Reposition. Er liegt in der Kreuzungs-
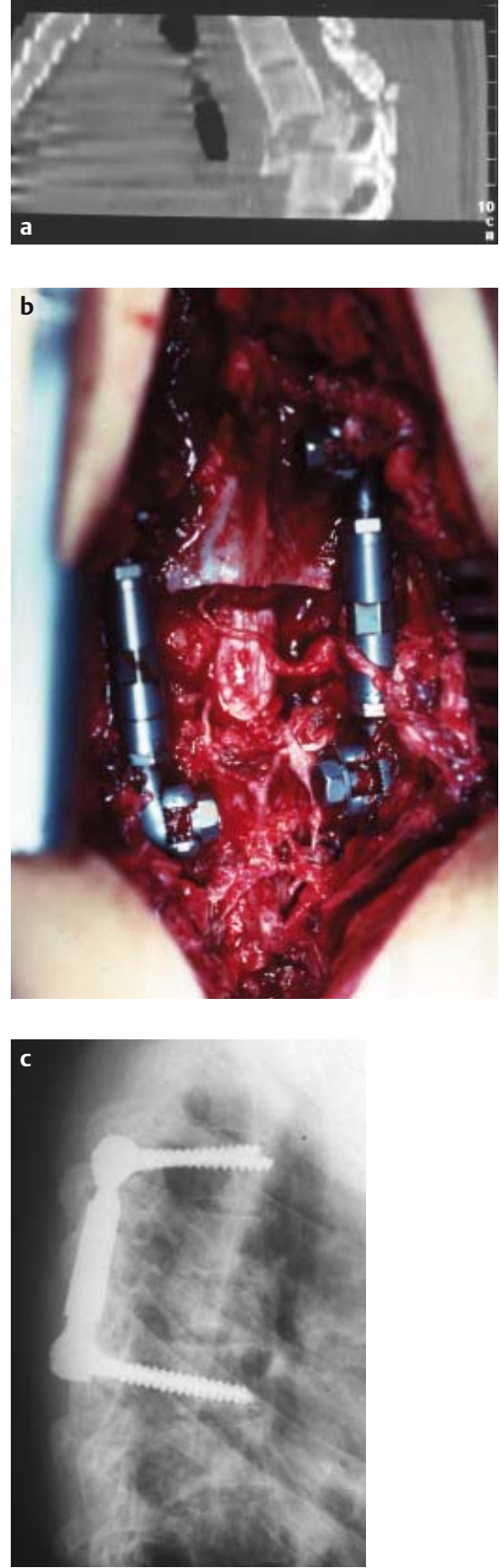

Abb.9a-c Flexions-Distraktions-Verletzung der BWS in Höhe von Th6. In a ist in der seitlichen CT-Rekonstruktion gut der nach ventral luxierte Wirbelkörper und der zerborstene Wirbelkörper Th6 zu erkennen. In b ist der intraoperative Situs nach Reposition und Instrumentation dargestellt. Gut erkennbar die traumatische Laminektomie mit freiliegender Dura mater. c zeigt die erreichte Wiederaufrichtung der Wirbelsäule und die Fixierung mit den Pedikelschrauben.

ebene Schanz-Schrauben/Längsträger hinter dem zerstörten Wirbelkörper. Durch Zusammendrücken der Verlängerungshebel der Schrauben dorsal erreicht 

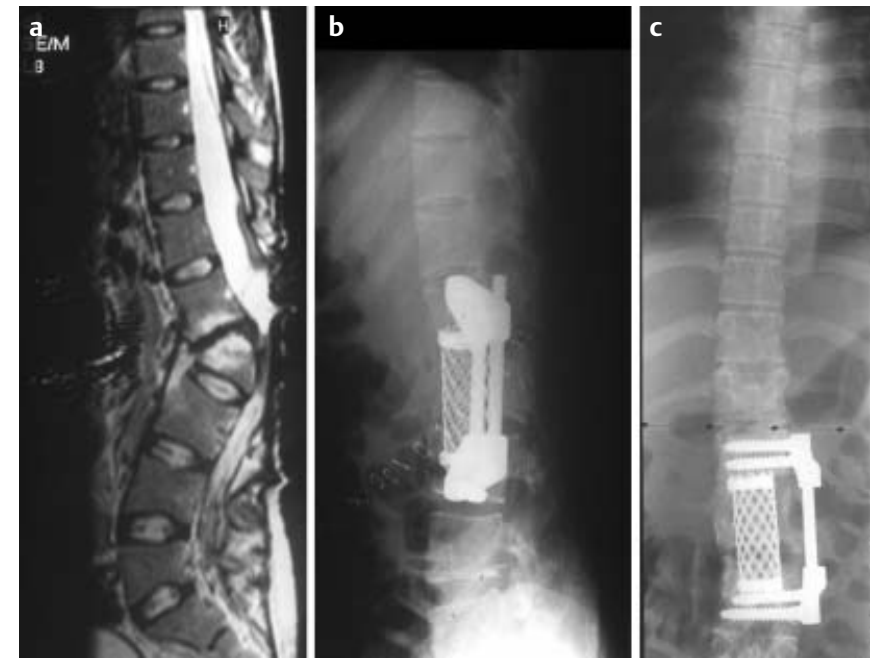

Abb.10a-c Z.n. ausgiebiger dorsaler Dekompression ohne Stabilisation nach LWK 2 Fraktur: a zeigt die sagittale Rekonstruktion des NMR-Bildes. Beachte die dünne dorsale Weichtaldeckung über dem Kyphosescheitel. bu. c zeigen den Zustand nach ventraler Aufrichtung der Wirbelsäule und Interposition eines Harms-Titangeflecht Korbes.

man gleichzeitig ventral eine Aufrichtung und Reposition. Verletzt man dieses Prinzip, lässt sich der Wirbelkörper nur ungenügend aufrichten und/oder es kommt zusätzlich zu einer Zugbelastung auf das Rückenmark. Abb. 8 zeigt schematisch das Prinzip.

Die Abb.9a-c zeigen exemplarisch die sagittale Rekonstruktion einer BWK 6Fraktur nach Motorradunfall, den intraoperativen Situs nach Implantation des Osteosynthesematerials und die postoperativ dokumentierte Wiederherstellung des sagittalen Profils der Wirbelsäule.

In keinem Falle akzeptabel ist nach einer Wirbelfraktur mit Rückenmarkschaden eine ausschließlich dorsale Dekompression durch Laminektomie. Abb.10a-c zeigt ein solches Beispiel. Bei der jungen Patientin ist nach einer LWK I-Verletzung eine großzügige Laminektomie durchgeführt worden. Die Lähmungssituation ist dadurch unverändert geblieben, jedoch ist die Wirbelsäule innerhalb von zwei Jahren in eine instabile kyphotische Fehlstellung abgerutscht. Diese Patientin war nicht mehr sitzfähig. Außerdem bestand die ständige Gefahr eines Dekubitus über dem Gibbusscheitel. Die Lähmungssituation war vor und nach der Operation komplett. Abb.10bu.c zeigen die notwendige, aufwändige ventrale Rekonstruktion, um Sitzfähigkeit zu erreichen.
Von zunehmender Bedeutung ist die minimale ventrale Stabilisation, die von T4 bis L1 regelmäßig zur Verfügung steht. Die Vorteile einer nur geringen Zerstörung der Brustwand und ihrer Muskulatur sind hoch $\mathrm{zu}$ bewerten. Allerdings muss die Technik sicher beherrscht werden. Die aus mangelnder Übung resultierenden OP-Zeiten mit hohem Blutverlust sind bei einer frischen Querschnittlähmung und den bereits oben angesprochenen Lungenproblemen nicht akzeptabel.

Sorgfältige Abwägung eines Notfalleingriffes, wenn möglich elektives, dem Frakturtyp angepasstes Vorgehen und weitest möglichem Wiederherstellen von Wirbelsäulenform und Spinalkanal.

\section{Schlussfolgerung}

Verletzungen der Wirbelsäule mit Querschnittlähmung sind schwere Verletzungen mit ausgedehnten Begleitschädigungen. Ihre Versorgung ist komplex, die Indikation zur notfallmäßigen operativen Stabilisation nur auf Einzelfälle beschränkt. Die Versorgung sollte deshalb in dafür spezialisierten Zentren und von Teams mit ausreichender Erfahrung erfolgen. Eine $24 \mathrm{~h}$ und 7 Tage die Woche besetzte Hotline an der BG-Unfallklinik Hamburg, (Tel. 040/ 7306-2604) gibt Auskunft und Hilfestellung und nennt die übernahmebereiten Zentren mit freier Bettenkapazität und Versorgungsmöglichkeit.

Das Schlusswort im beeindruckenden Werk von Wagner und Stolper [9] gilt noch nach mehr als 100 Jahren unverändert: „Die vertieftere Kenntnis der Verletzungen der Wirbelsäule und des Rückenmarks lehrt, dass man von einer sorgfältigen Behandlung für die knöcherne Wirbelsäule erheblich mehr erwarten darf, als man früher angenommen hat, für das Rückenmark aber entschieden weniger."

\section{Literatur}

1 Abel R, Gerner HJ, Smit C, Meiners T. Residual deformity of the spinal canal in patients with traumatic paraplegia and secondary changes of the spinal cord. Spina Cord 1999; 37: $14-19$

2 Boerger TO, Limb D, Dickson RA. Does canal claerance“ affect neurological outcome after thoracolumbar burst fractures? J Bone Joint Surg Br 2000; 82 (5): 629-35

3 Dick W. Innere Fixation von Brust- und Lendenwirbelfrakturen In: Burri C, Harder F, Jäger M. (Hrsg.) Aktuelle Probleme in Chirurgie und Orthopädie Bd. 28, Huber Bern Stuttgart Toronto, (1984)

4 Frankel H, Hancock HO, Hyslop G, Melzak J, Michaelis L, Un-gar G, Vernon J, Walsh J. The value of postural reduction in the initial management of closed injuries of the spine with paraplegia and tetraplegia. Paraplegie 1969; 7: 179-192

5 Gerner HJ. Die Querschnittlähmung - Erstversorgung, Behandlungstrategie, Rehabilitation, Blackwell Wissenschaft, Berlin (1992)

6 Guttmann L. Spinal cord injuries. 2nd edn. Oxford: Blackwell (1976)

7 Hughes JT. The Edwin Smith Surgical Papyrus: An analysis of the first case reports of spinal cord injuries. Paraple gia 1988; 26: $71-82$

8 Magerl F, Aebi M, Gertzbein SD, Harms J, Nazarian S. A comprehensive classification of thoracic an lumbar injuries. Eur Spine J. 1994; 3: 184-201

9 Wagner W, Stolper P. Die Verletzungen der Wirbelsäule und des Rückenmarks In: Bergmann E.v., Bruns P.v., Deutsche Chirurgie Enke Stuttgart, Bd 40 (1898)

\section{Dr. med. Rainer Abel}

Oberarzt

Prof. Dr. med. Hans Jürgen Gerner Ärztl. Direktor

Orthopädische Universitätsklinik Abteilung Orthopädie II

Schlierbacher Landstr. 200a

D-69118 Heidelberg 